\title{
ARTICLE
}

\section{A unified mechanism for proteolysis and autocatalytic activation in the $20 \mathrm{~S}$ proteasome}

Eva M. Huber ${ }^{1}$, Wolfgang Heinemeyer ${ }^{1}, \mathrm{Xia} \mathrm{Li}^{2, \dagger}{ }^{\dagger}$, Cassandra S. Arendt ${ }^{3, \dagger}$, Mark Hochstrasser ${ }^{2} \&$ Michael Groll $^{1}$

Biogenesis of the $20 \mathrm{~S}$ proteasome is tightly regulated. The $\mathrm{N}$-terminal propeptides protecting the active-site threonines are autocatalytically released only on completion of assembly. However, the trigger for the self-activation and the reason for the strict conservation of threonine as the active site nucleophile remain enigmatic. Here we use mutagenesis, $\mathrm{X}$-ray crystallography and biochemical assays to suggest that Lys 33 initiates nucleophilic attack of the propeptide by deprotonating the Thr1 hydroxyl group and that both residues together with Asp17 are part of a catalytic triad. Substitution of Thr1 by Cys disrupts the interaction with Lys33 and inactivates the proteasome. Although a Thr1Ser mutant is active, it is less efficient compared with wild type because of the unfavourable orientation of Ser1 towards incoming substrates. This work provides insights into the basic mechanism of proteolysis and propeptide autolysis, as well as the evolutionary pressures that drove the proteasome to become a threonine protease.

\footnotetext{
${ }^{1}$ Center for Integrated Protein Science at the Department Chemie, Lehrstuhl für Biochemie, Technische Universität München, Lichtenbergstrasse 4, 85747 Garching, Germany. ${ }^{2}$ Department of Molecular Biophysics and Biochemistry, Yale University, 266 Whitney Avenue, New Haven, Connecticut 06520-8114, USA. ${ }^{3}$ Department of Biochemistry and Molecular Biology, University of Chicago, Chicago, Illinois 60637, USA. †Present addresses: Department of Cellular and Molecular Medicine, UCSD, La Jolla, California 92093, USA (X.L.); 13417 Kinder Pass, Austin, Texas 78727, USA (C.S.A.). Correspondence and requests for materials should be addressed to E.M.H. (email: eva.huber@tum.de) or to M.G. (email: michael.groll@tum.de).
} 
T he $20 \mathrm{~S}$ proteasome core particle $(\mathrm{CP})$ is the key non-lysosomal protease of eukaryotic cells. Its seven different $\alpha$ and seven different $\beta$ subunits assemble into four heptameric rings that are stacked on each other to form a hollow cylinder. While the inactive $\alpha$ subunits build the two outer rings, the $\beta$ subunits form the inner rings. Only three out of the seven different $\beta$ subunits, namely $\beta 1, \beta 2$ and $\beta 5$, bear $\mathrm{N}$-terminal proteolytic active centres, and before $\mathrm{CP}$ maturation these are protected by propeptides ${ }^{1-3}$. In the last stage of CP biogenesis, the prosegments are autocatalytically removed through nucleophilic attack by the active site residue Thr1 on the preceding peptide bond involving Gly $(-1)^{4,5}$. Release of the propeptides creates a functionally active $\mathrm{CP}$ that cleaves proteins into short peptides.

Although the chemical nature of the substrate-binding channel and hence substrate preferences are unique to each of the distinct active $\beta$ subunits ${ }^{6,7}$, all active sites employ an identical reaction mechanism to hydrolyse peptide bonds ${ }^{2}$. Nucleophilic attack of $\mathrm{Thr} \mathrm{O}^{\gamma}$ on the carbonyl carbon atom of the scissile peptide bond creates a first cleavage product and a covalent acyl-enzyme intermediate. Hydrolysis of this complex by the addition of a nucleophilic water molecule regenerates the enzyme and releases the second peptide fragment ${ }^{8,9}$. The proteasome belongs to the family of N-terminal nucleophilic (Ntn) hydrolases ${ }^{10}$, and the free N-terminal amine group of Thrl was proposed to deprotonate the Thr1 hydroxyl group to generate a nucleophilic Thr1O $^{\gamma}$ for peptide-bond cleavage ${ }^{2,9,11}$. This mechanism, however, cannot explain autocatalytic precursor processing because in the immature active sites, ThrlN is part of the peptide bond with $\operatorname{Gly}(-1)$, the bond that needs to be hydrolysed. An alternative candidate for deprotonating the Thrl hydroxyl group is the side chain of Lys33 as it is within hydrogen-bonding distance to Thr1OH $(2.7 \AA)$. In principle it could function as the general base during both autocatalytic removal of the propeptide and protein substrate cleavage. Here we provide experimental evidences for this distinct view of the proteasome active-site mechanism. Data from biochemical and structural analyses of proteasome variants with mutations in the $\beta 5$ propeptide and the active site strongly support the model and deliver novel insights into the structural constraints required for the autocatalytic activation of the proteasome. Furthermore, we determine the advantages of Thr over Cys or Ser as the active-site nucleophile using X-ray crystallography together with activity and inhibition assays.

\section{Results}

Inactivation of proteasome subunits by T1A mutations. Proteasome-mediated degradation of cell-cycle regulators and potentially toxic misfolded proteins is required for the viability of eukaryotic cells ${ }^{8}$. Inactivation of the active site Thr1 by mutation to Ala has been used to study substrate specificity and the hierarchy of the proteasome active sites $1,4,12-15$. Yeast strains carrying the single mutations $\beta 1-\mathrm{T} 1 \mathrm{~A}$ or $\beta 2-\mathrm{T} 1 \mathrm{~A}$, or both, are viable, even though one or two of the three distinct catalytic $\beta$ subunits are disabled and carry remnants of their N-terminal propeptides $^{4}$ (Table 1). These results indicate that the $\beta 1$ and $\beta 2$ proteolytic activities are not essential for cell survival. By contrast, the T1A mutation in subunit $\beta 5$ has been reported to be lethal or nearly so ${ }^{1,13}$. Viability is restored if the $\beta 5$-T1A subunit has its propeptide (pp) deleted but expressed separately in trans ( $\beta 5$-T1A pp trans), although substantial phenotypic impairment remains $1,15,16$ (Table 1). Our present crystallographic analysis of the $\beta 5$-T1A pp trans mutant demonstrates that the mutation per se does not structurally alter the catalytic active site and that the trans-expressed $\beta 5$ propeptide is not bound in the $\beta 5$ substrate-binding channel (Supplementary Fig. 1a).
The extremely weak growth of the $\beta 5$-T1A mutant pp cis described by Chen and Hochstrasser ${ }^{1}$ compared with the inviability reported by Heinemeyer et al. ${ }^{13}$ prompted us to analyse this discrepancy. Sequencing of the plasmids, testing them in both published yeast strain backgrounds and site-directed mutagenesis revealed that the $\beta 5$-T1A mutant $\mathrm{pp}$ cis is viable, but suffers from a marked growth defect that requires extended incubation of 4-5 days for initial colony formation (Table 1 and Supplementary Methods). We also identified an additional point mutation K81R in subunit $\beta 5$ that was present in the allele used in ref. 1 . This single amino-acid exchange is located at the interface of the subunits $\alpha 4, \beta 4$ and $\beta 5$ (Supplementary Fig. 1b) and might weakly promote $\mathrm{CP}$ assembly by enhancing intersubunit contacts. The slightly better growth of the 35 -T1A-K81R mutant allowed us to solve the crystal structure of a yeast proteasome (yCP) with the $\beta 5$-T1A mutation, which is discussed in the following section (for details see Supplementary Note 1).

Propeptide conformation and triggering of autolysis. In the final steps of proteasome biogenesis, the propeptides are autocatalytically cleaved from the mature $\beta$-subunit domains ${ }^{1}$. For subunit $\beta 1$, this process was previously inferred to require that the propeptide residue at position $(-2)$ of the subunit precursor occupies the S1 specificity pocket of the substratebinding channel formed by amino acid 45 (for details see Supplementary Note 2$)^{5}$. Furthermore, it was observed that the prosegment forms an antiparallel $\beta$-sheet in the active site, and that Gly(-1) adopts a $\gamma$-turn conformation, which by definition is characterized by a hydrogen bond between Leu(-2)O and Thr1NH (ref. 5). Here we again analysed the $\beta 1$-T1A mutant crystallographically but in addition determined the structures of the $\beta 2$-T1A single and $\beta 1$-T1A- $\beta 2-T 1 A$ double mutants (Protein Data Bank (PDB) entry codes are provided in Supplementary Table 1$)$. In subunit $\beta 1$, we found that $\operatorname{Gly}(-1)$ indeed forms a sharp turn, which relaxes on prosegment cleavage (Fig. 1a and Supplementary Fig. 2a). However, the $\gamma$-turn conformation and the associated hydrogen bond initially proposed is for geometric and chemical reasons inappropriate and would not perfectly position the carbonyl carbon atom of Gly(-1) for nucleophilic attack by Thr1. Regarding the $\beta 2$ propeptide, $\operatorname{Thr}(-2)$ occupies the $\mathrm{S} 1$ pocket but is less deeply anchored compared with Leu(-2) in $\beta 1$, which might be due to the rather large $\beta 2-S 1$ pocket created by Gly45. Thr(-2) positions Gly $(-1) \mathrm{O}$ via hydrogen bonding $(\sim 2.8 \AA)$ in a perfect trajectory for the nucleophilic attack by $\mathrm{Thr}^{\gamma} \mathrm{O}^{\gamma}$ (Fig. $1 \mathrm{~b}$ and Supplementary Fig. $2 \mathrm{~b}$ ). Next, we examined the position of the $\beta 5$ propeptide in the $\beta 5-T 1 A-K 81 R$ mutant. Surprisingly, Gly $(-1)$ is completely extended and forces the histidine side chain at position (-2) to occupy the S2 instead of the S1 pocket, thereby disrupting the antiparallel $\beta$-sheet. Nonetheless, the carbonyl carbon of Gly $(-1)$ would be ideally placed for nucleophilic attack by $\mathrm{Thr}^{\gamma} \mathrm{O}^{\gamma}$ (Fig. 1c and Supplementary Fig. 2c,d). As the K81R mutation is located far from the active site (Thr1C $\left.{ }^{\alpha}-\operatorname{Arg} 81 C^{\alpha}: 24 \AA\right)$, any influence on propeptide conformation can be excluded. Instead, the plasticity of the $\beta 5 \mathrm{~S} 1$ pocket caused by the rotational flexibility of Met45 might prevent stable accommodation of His(-2) in the S1 site and thus also promote its immediate release after autolysis.

Processing of $\beta$-subunit precursors requires deprotonation of Thr1OH; however, the general base initiating autolysis is unknown. Remarkably, eukaryotic proteasomal $\beta 5$ subunits bear a His residue in position (-2) of the propeptide (Supplementary Fig. 3a). As histidine commonly functions as a proton shuttle in the catalytic triads of serine proteases ${ }^{17}$, we investigated the role of His(-2) in processing of the $\beta 5$ propeptide by exchanging it for Asn, Lys, Phe and Ala. All yeast mutants were viable at $30^{\circ} \mathrm{C}$, 
Table 1 | Growth phenotypes and status of autolysis and catalysis of mutants.

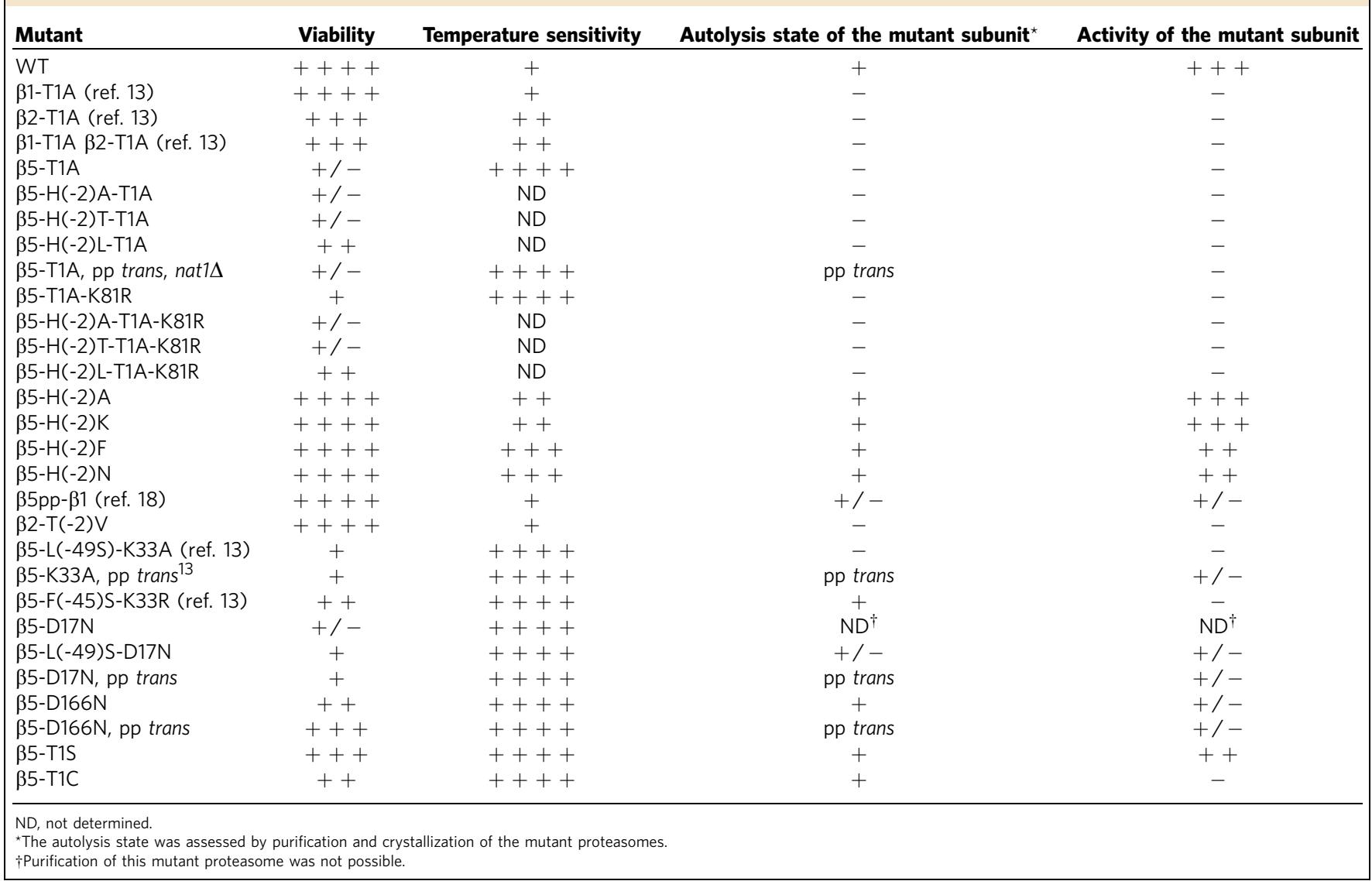

a

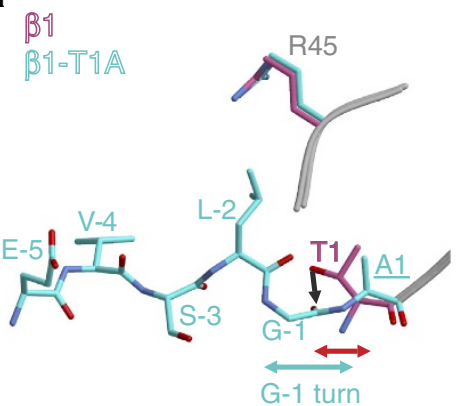

b

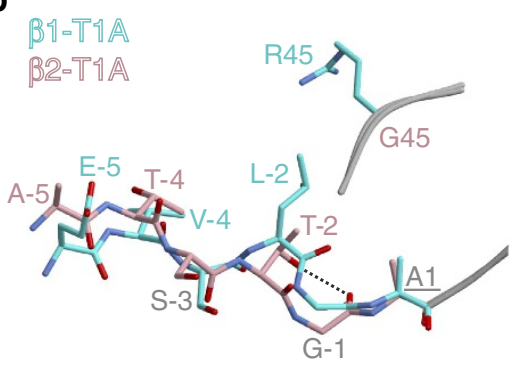

C

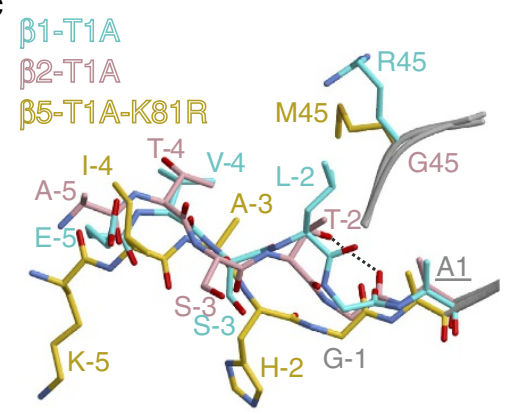

Figure 1 | Conformation of proteasomal propeptides. (a) Structural superposition of the $\beta 1-T 1 A$ propeptide and the matured WT $\beta 1$ active-site Thr1. Only the residues $(-5)$ to $(-1)$ of the $\beta 1-$ T1A propeptide are displayed. The major determinant of the S1 specificity pocket, residue 45 , is depicted. Note the tight conformation of Gly $(-1)$ and Ala1 before propeptide removal (G(-1) turn; cyan double arrow) compared with the relaxed, processed WT active-site Thr1 (red double arrow). The black arrow indicates the attack of Thr1O $\gamma$ onto the carbonyl carbon atom of Gly(-1). (b) Structural superposition of the $\beta 1-T 1 A$ propeptide and the $\beta 2-$ T1A propeptide highlights subtle differences in their conformations, but illustrates that Ala1 and Gly(-1) match well. Thr(-2)OH is hydrogen-bonded to Gly(-1)O ( $\sim 2.8 \AA$; black dashed line). The major determinant of the S1 specificity pocket, residue 45 , is depicted. (c) Structural superposition of the $\beta 1-T 1 A$, the $\beta 2-$ T1A and the $\beta 5-T 1 A-K 81 R$ propeptide remnants depict their differences in conformation. While residue (-2) of the $\beta 1$ and $\beta 2$ prosegments fit the S1 pocket, His $(-2)$ of the $\beta 5$ propeptide occupies the S2 pocket. Nonetheless, in all mutants the carbonyl carbon atom of Gly $(-1)$ is ideally placed for the nucleophilic attack by Thr10\%. The hydrogen bond between $\operatorname{Thr}(-2) \mathrm{OH}$ and Gly $(-1) \mathrm{O}(\sim 2.8 \AA)$ is indicated by a black dashed line.

but suffered from growth defects at $37^{\circ} \mathrm{C}$ with the $\mathrm{H}(-2) \mathrm{N}$ and $\mathrm{H}(-2) \mathrm{F}$ mutants being most affected (Supplementary Fig. $3 \mathrm{~b}$ and Table 1). In agreement, the chymotrypsin-like (ChT-L) activity of $\mathrm{H}(-2) \mathrm{N}$ and $\mathrm{H}(-2) \mathrm{F}$ mutant yCPs was impaired in situ and in vitro (Supplementary Fig. 3c). Structural analyses revealed that the propeptides of all mutant yCPs shared residual $2 F_{\mathrm{O}}-F_{\mathrm{C}}$ electron densities. Gly(-1) and Phe/Lys(-2) were visualized at low occupancy, while Ala/Asn(-2) could not be assigned. This observation indicates a mixture of processed and unprocessed $\beta 5$ subunits and partially impaired autolysis ${ }^{18}$, thereby excluding any essential role of residue $(-2)$ as the general base.

Next, we examined the effect of residue $(-2)$ on the orientation of the propeptide by creating mutants that combine the T1A (K81R) mutation(s) with $\mathrm{H}(-2) \mathrm{L}, \mathrm{H}(-2) \mathrm{T}$ or $\mathrm{H}(-2) \mathrm{A}$ substitutions. Leu(-2) is encoded in the yeast $\beta 1$ subunit precursor (Supplementary Fig. 3a); Thr(-2) is generally part of 
a

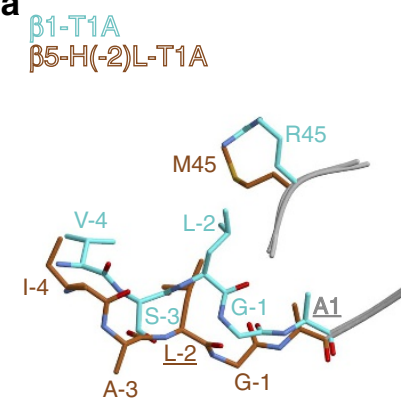

c

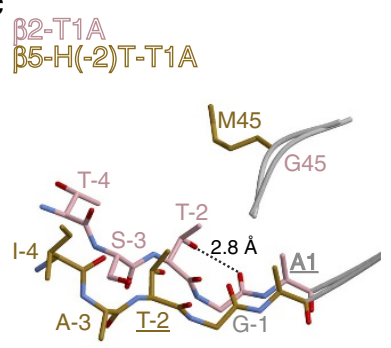

b

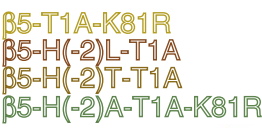

B5-H(-2) A-T1 A-K81R

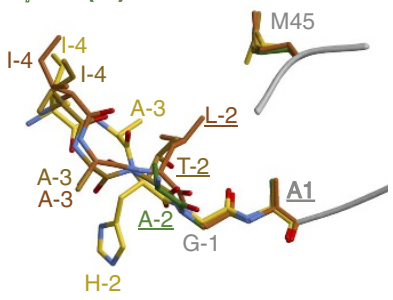

d

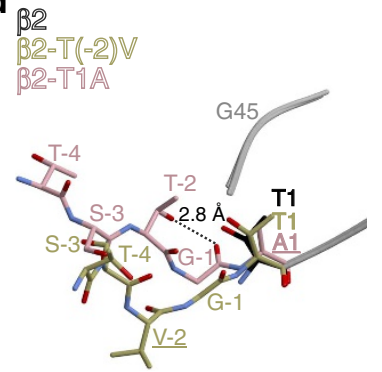

Figure 2 | Mutations of residue (-2) and their influence on propeptide conformation and autolysis. (a) Structural superposition of the $\beta 1-\mathrm{T} 1 \mathrm{~A}$ propeptide and the $\beta 5-H(-2) L-T 1 A$ mutant propeptide. The $(-2)$ residues of both prosegments point into the S1 pocket. (b) Structural superposition of the $\beta 5$ propeptides in the $\beta 5-\mathrm{H}(-2) \mathrm{L}-\mathrm{T} 1 \mathrm{~A}, \beta 5-\mathrm{H}(-2) \mathrm{T}-\mathrm{T} 1 \mathrm{~A}, \beta 5-(\mathrm{H}-2) \mathrm{A}-\mathrm{T} 1 \mathrm{~A}-$ K81R and $\beta 5-T 1 A-K 81 R$ mutant proteasomes. While the residues $(-2)$ to $(-4)$ vary in their conformation, Gly $(-1)$ and Ala1 are located in all structures at the same positions. (c) Structural superposition of the $\beta 2-\mathrm{T} 1 \mathrm{~A}$ propeptide and the $\beta 5-\mathrm{H}(-2) \mathrm{T}-\mathrm{T} 1 \mathrm{~A}$ mutant propeptide. The $(-2)$ residues of both prosegments point into the $\mathrm{S} 1$ pocket, but only $\operatorname{Thr}(-2) \mathrm{OH}$ of $\beta 2$ forms a hydrogen bridge to Gly(-1)O (black dashed line). (d) Structural superposition of the matured $\beta 2$ active site, the WT $\beta 2-$ T1A propeptide and the $\beta 2-\mathrm{T}(-2) \bigvee$ mutant propeptide. Notably, $\mathrm{Val}(-2)$ of the latter does not occupy the S1 pocket, thereby changing the orientation of Gly $(-1)$ and preventing nucleophilic attack of $\mathrm{Thr}^{1} \mathrm{O}^{\gamma}$ on the carbonyl carbon atom of Gly(-1). For all panels stereo views are provided in Supplementary Fig. $4 \mathrm{~g}-\mathrm{j}$.

$\beta 2$-propeptides (Supplementary Fig. 3a); and $\mathrm{Ala}(-2)$ was expected to fit the $\beta 5-S 1$ pocket without inducing conformational changes of Met45, allowing it to accommodate ' $\beta 1$-like' propeptide positioning. As expected from $\beta 5$-T1A mutants, the yeasts show severe growth phenotypes, with minor variations (Supplementary Fig. 4a and Table 1). We determined crystal structures of the $\beta 5-\mathrm{H}(-2) \mathrm{L}-\mathrm{T} 1 \mathrm{~A}, \beta 5-\mathrm{H}(-2) \mathrm{T}-\mathrm{T} 1 \mathrm{~A}$ and the $\beta 5-\mathrm{H}(-$ 2)A-T1A-K81R mutants (Supplementary Table 1). For the $\beta 5-\mathrm{H}(-2) \mathrm{A}-\mathrm{T} 1 \mathrm{~A}-\mathrm{K} 81 \mathrm{R}$ variant, only the residues $\mathrm{Gly}(-1)$ and $\mathrm{Ala}(-2)$ could be visualized, indicating that $\mathrm{Ala}(-2)$ leads to insufficient stabilization of the propeptide in the substratebinding channel (Supplementary Fig. 4d). By contrast, the prosegments of the $\beta 5-\mathrm{H}(-2) \mathrm{L}-\mathrm{T} 1 \mathrm{~A}$ and the $\beta 5-\mathrm{H}(-2) \mathrm{T}-\mathrm{T} 1 \mathrm{~A}$ mutants were significantly better resolved in the $2 F_{\mathrm{O}}-F_{\mathrm{C}}$ electrondensity maps yet not at full occupancy (Supplementary Fig. 4b,c and Supplementary Table 1), suggesting that the natural propeptide bearing His(-2) is most favourable. Nevertheless, both Leu(-2) and $\operatorname{Thr}(-2)$ were found to occupy the S1 specificity pocket formed by Met45 (Fig. 2a,b and Supplementary Fig. 4f-h). This result proves that the naturally occurring His $(-2)$ of the $\beta 5$ propeptide does not stably fit into the S1 site. Since Gly(-1) adopts the same position in both wild-type (WT) and mutant $\beta 5$ propeptides, and since in all cases its carbonyl carbon is perfectly placed for nucleophilic attack by $\mathrm{Thr}^{\gamma} \mathrm{O}^{\gamma}$ (Fig. 2b), we propose

that neither binding of residue $(-2)$ to the S1 pocket nor formation of the antiparallel $\beta$-sheet is essential for autolysis of the propeptide.

Next, we determined the crystal structure of a chimeric yCP having the yeast $\beta 1$-propeptide replaced by its $\beta 5$ counterpart $^{18}$. Although we observed fragments of $2 F_{\mathrm{O}}-F_{\mathrm{C}}$ electron density in the $\beta 1$ active site, the data were not interpretable. Bearing in mind that in contrast to $\operatorname{Thr}(-2)$ in $\beta 2$, Leu(-2) in subunit $\beta 1$ is not conserved among species (Supplementary Fig. 3a), we created a $\beta 2-\mathrm{T}(-2) \mathrm{V}$ proteasome mutant. As proven by the $\beta 2-\mathrm{T} 1 \mathrm{~A}$ crystal structures, $\operatorname{Thr}(-2)$ hydrogen bonds to $\mathrm{Gly}(-1) \mathrm{O}$. Although this interaction was not observed for the $\beta 5-\mathrm{H}(-2) \mathrm{T}-\mathrm{T} 1 \mathrm{~A}$ mutant (Fig. 2c and Supplementary Fig. 4c,i), exchange of Thr(-2) by Val in $\beta 2$, a conservative mutation regarding size but drastic with respect to polarity, was found to inhibit maturation of this subunit (Fig. $2 \mathrm{~d}$ and Supplementary Fig. $4 \mathrm{e}, \mathrm{j}$ ). Notably, the $2 \mathrm{~F}_{\mathrm{O}^{-}}$ $F_{\mathrm{C}}$ electron-density map displays a different orientation for the $\beta 2$ propeptide than has been observed for the $\beta 2$-T1A proteasome. In particular, $\operatorname{Val}(-2)$ is displaced from the $\mathrm{S} 1$ site and $\operatorname{Gly}(-1)$ is severely shifted (movement of the carbonyl oxygen atom of $3.8 \AA$ ), thereby preventing nucleophilic attack of Thr1 (Fig. 2d and Supplementary Fig. $4 \mathrm{j}, \mathrm{k})$. These results further confirm that correct positioning of the active-site residues and Gly(-1) is decisive for the maturation of the proteasome.

The active site of the proteasome. Proton shuttling from the proteasomal active site $\mathrm{Thr} 1 \mathrm{OH}$ to $\mathrm{Thr} 1 \mathrm{NH}_{2}$ via a nucleophilic water molecule was suggested to initiate peptide-bond hydrolysis ${ }^{2,9,10}$. However, in the immature particle $\mathrm{Thr}_{1 \mathrm{NH}}$ is blocked by the propeptide and cannot activate $\mathrm{Thr}^{\mathrm{O}} \mathrm{O}^{\gamma}$. Instead, Lys33 $\mathrm{NH}_{2}$, which is in hydrogen-bonding distance to $\mathrm{Thr}^{2} \mathrm{O}^{\gamma}$ $(2.7 \AA)$ in all catalytically active $\beta$ subunits (Fig. 3a,b) ${ }^{2,9}$, was proposed to serve as the proton acceptor ${ }^{19}$. Owing to its likely protonation at neutral $\mathrm{pH}$, however, it was assumed not to act as the general base ${ }^{2,5,9}$. A proposed catalytic tetrad model involving Thr1OH, Thr1 $1 \mathrm{NH}_{2}, \operatorname{Lys} 33 \mathrm{NH}_{2}$ and $A s p 17 \mathrm{O}^{\delta}$, as well as a nucleophilic water molecule as the proton shuttle appeared to accommodate all possible views of the proteasomal active site $^{8,9,20}$. Twenty years later, with a plethora of yCP X-ray structures in hand, we decided to re-analyse the active site of the proteasome and to resolve the uncertainty regarding the nature of the general base. Mutation of $\beta 5$-Lys33 to Ala causes a strongly deleterious phenotype, and previous structural and biochemical analyses confirmed that this is caused by failure of propeptide cleavage, and consequently, lack of ChT-L activity ${ }^{1,4,13}$ (Fig. 4a, Supplementary Fig. $3 \mathrm{~b}$ and Table 1; for details see Supplementary Note 1$)$. The phenotype of the $\beta 5$-K33A mutant was however less pronounced than for the $\beta 5-\mathrm{T} 1 \mathrm{~A}-\mathrm{K} 81 \mathrm{R}$ yeast (Fig. $4 \mathrm{a}$ ). This discrepancy in growth was traced to an additional point mutation $\mathrm{L}(-49) \mathrm{S}$ in the $\beta 5$-propeptide of the $\beta 5$-K33A mutant (see also Supplementary Note 1$)$. Structural comparison of the $\beta 5-\mathrm{L}(-49) \mathrm{S}$ $\mathrm{K} 33 \mathrm{~A}$ and $\beta 5-\mathrm{T} 1 \mathrm{~A}-\mathrm{K} 81 \mathrm{R}$ active sites revealed that mutation of Lys33 to Ala creates a cavity that is filled with Thr1 and the remnant propeptide. This structural alteration destroys active-site integrity and abolishes catalytic activity of the $\beta 5$ active site 4 (Supplementary Fig. 5a). Additional proof for the key function of Lys33 was obtained from the $\beta 5-\mathrm{K} 33 \mathrm{~A}$ mutant, with the propeptide expressed separately from the main subunit (pp trans) ${ }^{15}$. The Thr1 $\mathrm{N}$ terminus of this mutant is not blocked by the propeptide, yet its catalytic activity is reduced by $\sim 83 \%$ (Supplementary Fig. 6b). Consistent with this, the crystal structure of the $\beta 5-\mathrm{K} 33 \mathrm{~A}$ pp trans mutant in complex with carfilzomib only showed partial occupancy of the ligand at the $\beta 5$ active sites (Supplementary Fig. 5b and Supplementary Table 1). Since no acetylation of the Thr1 $\mathrm{N}$ terminus was observed for the 
a

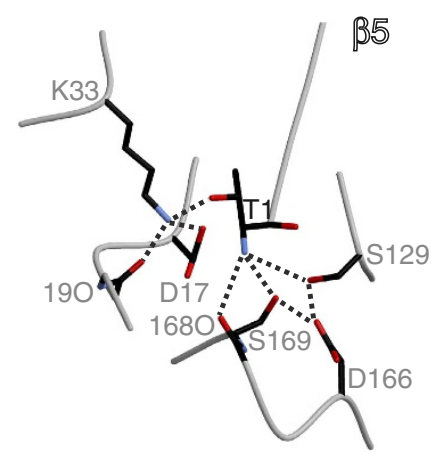

b

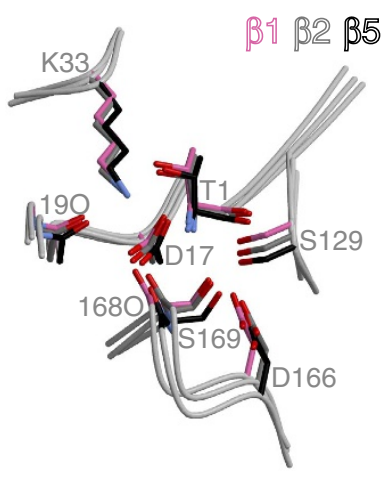

C

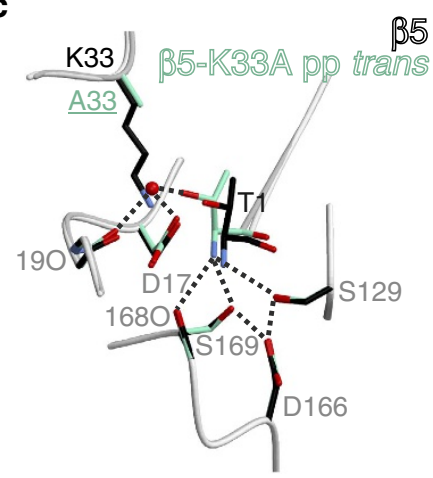

\section{Catalysis}

d

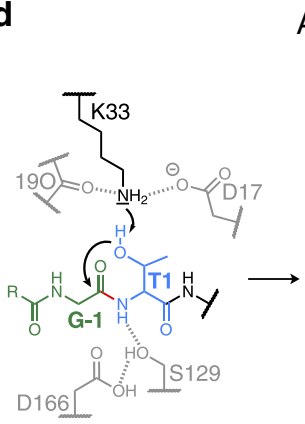

Autolysis

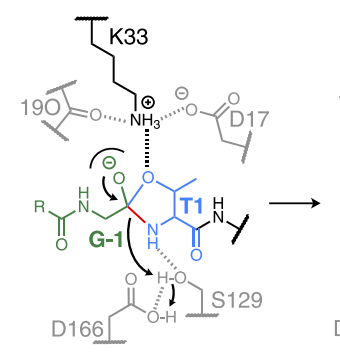

Hydroxyoxazolidine ring

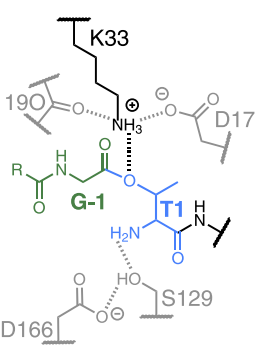

$\downarrow+\mathrm{H}_{2} \mathrm{O}$

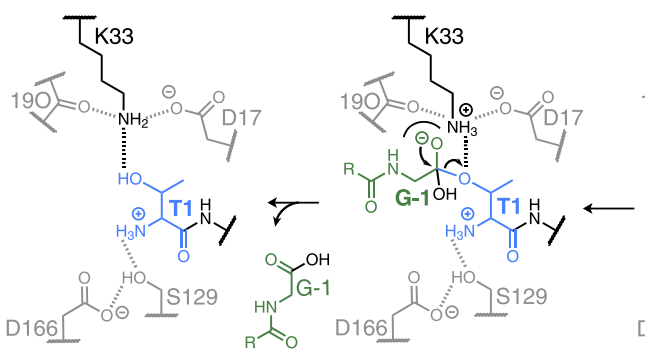

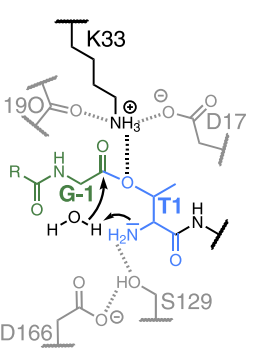

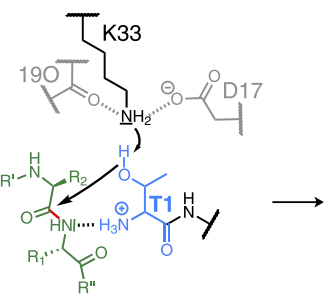

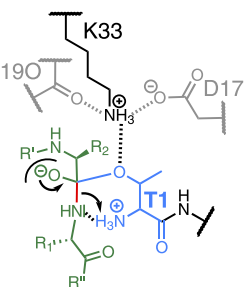

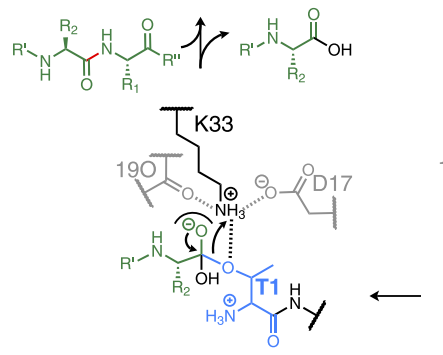

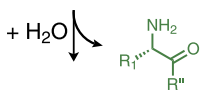

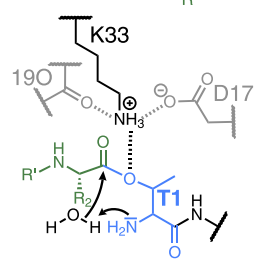

Figure 3 | Architecture and proposed reaction mechanism of the proteasomal active site. (a) Hydrogen-bonding network at the mature WT $\beta 5$ proteasomal active site (dotted lines). Thr1OH is hydrogen-bonded to Lys $33 \mathrm{NH}_{2}\left(2.7 \AA\right.$ ), which in turn interacts with $A s p 17 \mathrm{O}^{\delta}$. The Thr1 $\mathrm{N}$ terminus is engaged in hydrogen bonds with Ser1290 $\gamma$, the carbonyl oxygen of residue 168 , Ser1690 $\gamma$ and Asp $1660^{\delta}$. (b) The orientations of the active-site residues involved in hydrogen bonding are strictly conserved in each proteolytic centre, as shown by superposition of the $\beta$ subunits. (c) Structural superposition of the WT $\beta 5$ and the $35-\mathrm{K} 33 \mathrm{~A}$ pp trans mutant active site. In the latter, a water molecule (red sphere) is found at the position where in the WT structure the side chain amine group of Lys33 is located. Similarly to Lys33, the water molecule hydrogen bonds to Arg190, Asp170 $\delta$ and Thr1OH. Note, the strong interaction with the water molecule causes a minor shift of Thr1, while all other active-site residues remain in place. (d) Proposed chemical reaction mechanism for autocatalytic precursor processing and proteolysis in the proteasome. The active-site Thr 1 is depicted in blue, the propeptide segment and the peptide substrate are coloured in green, whereas the scissile peptide bond is highlighted in red. Autolysis (left set of structures) is initiated by deprotonation of $\mathrm{Thr} 1 \mathrm{OH}$ via $\mathrm{Lys} 33 \mathrm{NH}_{2}$ and the formation of a tetrahedral transition state. The strictly conserved oxyanion hole Gly47NH stabilizing the negatively charged intermediate is illustrated as a semicircle. Collapse of the transition state frees the Thr1 $\mathrm{N}$ terminus (by completing an $\mathrm{N}$-to-O acyl shift of the propeptide), which is subsequently protonated by $\mathrm{Asp} 166 \mathrm{OH}$ via Ser129OH. Next, Thr1 $\mathrm{NH}_{2}$ polarizes a water molecule for the nucleophilic attack of the acyl-enzyme intermediate. On hydrolysis of the latter, the active-site Thr1 is ready for catalysis (right set of structures). Substrate processing starts with nucleophilic attack of the carbonyl carbon atom of the scissile peptide bond. The charged Thr1 $\mathrm{N}$ terminus may engage in the orientation of the amide moiety and donate a proton to the emerging $\mathrm{N}$ terminus of the $\mathrm{C}$-terminal cleavage product. The resulting deprotonated Thr $1 \mathrm{NH}_{2}$ finally activates a water molecule for hydrolysis of the acyl-enzyme.

35-K33A pp trans apo crystal structure ${ }^{4,16}$, the reduced reactivity towards substrates and inhibitors indicates that $\mathrm{Lys} 33 \mathrm{NH}_{2}$, rather than $\mathrm{Thr} 1 \mathrm{NH}_{2}$, deprotonates and activates $\mathrm{Thr} 1 \mathrm{OH}$. Furthermore, the crystal structure of the $\beta 5-\mathrm{K} 33 \mathrm{~A}$ pp trans mutant without inhibitor revealed that $\mathrm{Thr}^{\gamma} \mathrm{O}^{\gamma}$ strongly coordinates a well-defined water molecule ( $\sim 2 \AA$; Fig. $3 c$ and Supplementary Fig. 5 c,d). This water hydrogen bonds also to

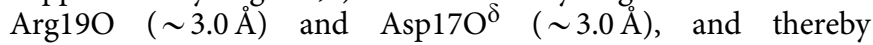

presumably enables residual activity of the mutant. Remarkably, the solvent molecule occupies the position normally taken by Lys $33 \mathrm{NH}_{2}$ in the WT proteasome structure (Fig. 3c), further corroborating the essential role of Lys 33 as the general base for autolysis and proteolysis. Conservative substitution of Lys 33 by Arg delays autolysis of the $\beta 5$ precursor and impairs yeast growth (for details see Supplementary Note 1). While Thr1 occupies the same position as in WT yCPs, Arg33 is unable to hydrogen 
bond to Asp17, thereby inactivating the $\beta 5$ active site 2,4 (Supplementary Fig. 5e).

The conservative mutation of Asp17 to Asn in subunit $\beta 5$ of the yCP also provokes a severe growth defect (Supplementary Note 1, Supplementary Fig. 6a and Table 1). Notably, only with the additional point mutation $\mathrm{L}(-49) \mathrm{S}$ present in the $\beta 5$ propeptide could we purify a small amount of the $\beta 5-\mathrm{D} 17 \mathrm{~N}$ mutant yCP. As determined by crystallographic analysis, this mutant $\beta 5$ subunit was partially processed (Table 1 ) but displayed impaired reactivity towards the proteasome inhibitor carfilzomib compared with the subunits $\beta 1$ and $\beta 2$, and with WT $\beta 5$ (Supplementary Fig. 7a). In contrast to the cis-construct, expression of the $\beta 5$ propeptide in trans allowed straightforward isolation and crystallization of the D17N mutant proteasome. The ChT-L activity of the $\beta 5-\mathrm{D} 17 \mathrm{~N}$ pp in trans CP towards the canonical $\beta 5$ model substrates $N$-succinyl-Leu-Leu-Val-Tyr7-amino-4-methylcoumarin (Suc-LLVY-AMC) and carboxybenzyl-Gly-Gly-Leu-para-nitroanilide (Z-GGL-pNA) was severely reduced (Supplementary Fig. 6b), confirming that Asp17 is of fundamental importance for the catalytic activity of the mature proteasome. Even though the $\beta 5-\mathrm{D} 17 \mathrm{~N}$ pp trans yCP crystal structure appeared identical to the WT yCP (Supplementary Fig. 7b), the co-crystal structure with the $\alpha^{\prime}, \beta^{\prime}$ epoxyketone inhibitor carfilzomib visualized only partial occupancy of the ligand in the $\beta 5$ active site (Supplementary Fig. 7a). This observation is consistent with a strongly reduced reactivity of $\beta 5-\mathrm{Thr} 1$ and the crystal structure of the $\beta 5-\mathrm{D} 17 \mathrm{~N}$ pp cis mutant in complex with carfilzomib. Autolysis and residual catalytic activity of the $\beta 5-\mathrm{D} 17 \mathrm{~N}$ mutants may originate from the carbonyl group of Asn17, which albeit to a lower degree still can polarize Lys33 for the activation of Thr1. In agreement, an E17A mutant in the proteasomal $\beta$-subunit of the archaeon Thermoplasma acidophilum prevents autolysis and catalysis ${ }^{21}$. Strikingly, although the X-ray data on the $\beta 5-\mathrm{D} 17 \mathrm{~N}$ mutant with the propeptide expressed in cis and in trans looked similar, there was a pronounced difference in their growth phenotypes observed (Supplementary Fig. 6a and Supplementary Fig. 7b).

On the basis of these results, we propose that CPs from all domains of life use a catalytic triad consisting of Thr1, Lys33 and Asp/Glu17 for both autocatalytic precursor processing and proteolysis (Fig. 3d). This model is also consistent with the fact that no defined water molecule is observed in the mature WT proteasomal active site that could shuttle the proton from $\mathrm{Thr} \mathrm{O}^{\gamma}$ to $\mathrm{Thr}_{1} \mathrm{NH}_{2}$.

To explore this active-site model further, we exchanged the conserved Asp166 residue for Asn in the yeast $\beta 5$ subunit. Asp $166 \mathrm{O}^{\delta}$ is hydrogen-bonded to $\mathrm{Thr} 1 \mathrm{NH}_{2}$ via Ser129OH and Ser169OH, and therefore was proposed to be involved in catalysis $^{2}$. The $\beta 5-\mathrm{D} 166 \mathrm{~N}$ pp cis yeast mutant is significantly impaired in growth and its ChT-L activity is drastically reduced (Supplementary Fig. 6a,b and Table 1). X-ray data on the $\beta 5-\mathrm{D} 166 \mathrm{~N}$ mutant indicate that the $\beta 5$ propeptide is hydrolysed, but due to reorientation of Ser $1290 \mathrm{OH}$, the interaction with Asn $166 \mathrm{O}^{\delta}$ is disrupted (Supplementary Fig. 8a). Instead, a water molecule is bound to Ser129OH and $\mathrm{Thr} \mathrm{NH}_{2}$ (Supplementary Fig. $8 \mathrm{~b}$ ), which may enable precursor processing. The hydrogen bonds involving Ser169OH are intact and may account for residual substrate turnover. Soaking the $\beta 5-\mathrm{D} 166 \mathrm{~N}$ crystals with carfilzomib and MG132 resulted in covalent modification of Thr1 at high occupancy (Supplementary Fig. 8c). In the carfilzomib complex structure, Thr $1 \mathrm{O}^{\gamma}$ and Thr1N incorporate into a morpholine ring structure and Ser129 adopts its WT-like orientation. In the MG132-bound state, Thr1N is unmodified, and we again observe that Ser129 is hydrogen-bonded to a water molecule instead of Asn166. Whereas Asn can to some degree replace Asp166 due to its carbonyl group in the side chain, Ala at this position was found to prevent both autolysis and catalysis ${ }^{21}$. These results suggest that Asp166 and Ser129 function as a proton shuttle and affect the protonation state of Thr1N during autolysis and catalysis.

Substitution of the active-site Thr1 by Cys. Mutation of Thr1 to Cys inactivates the $20 \mathrm{~S}$ proteasome from the archaeon T. acidophilum ${ }^{21}$. In yeast, this mutation causes a strong growth defect (Fig. 4a and Table 1), although the propeptide is hydrolysed, as shown here by its X-ray structure. In one of the two $\beta 5$ subunits, however, we found the cleaved propeptide still bound in the substrate-binding channel (Fig. 4c). His(-2) occupies the S2 pocket like observed for the $\beta 5-\mathrm{T} 1 \mathrm{~A}-\mathrm{K} 81 \mathrm{R}$ mutant, but in contrast to the latter, the propeptide in the T1C mutant adopts an antiparallel $\beta$-sheet conformation as known from inhibitors like MG132 (Fig. 4c-e and Supplementary Fig. 9b). On the basis of the phenotype of the T1C mutant and the propeptide remnant identified in its active site, we suppose that autolysis is retarded and may not have been completed before crystallization. Owing to the unequal positions of the two $\beta 5$ subunits within the CP in the crystal lattice, maturation and propeptide displacement may occur at different timescales in the two subunits.

Despite propeptide hydrolysis, the $\beta 5-\mathrm{T} 1 \mathrm{C}$ active site is catalytically inactive (Fig. $4 \mathrm{~b}$ and Supplementary Fig. 9a). In agreement, soaking crystals with the $\mathrm{CP}$ inhibitors bortezomib or carfilzomib modifies only the $\beta 1$ and $\beta 2$ active sites, while leaving the $\beta 5$-T1C proteolytic centres unmodified even though they are only partially occupied by the cleaved propeptide remnant. Moreover, the structural data reveal that the thiol group of Cys1 is rotated by $74^{\circ}$ with respect to the hydroxyl side chain of Thr1 (Fig. 4f and Supplementary Fig. 9b). This presumably results from the larger radius of the sulfur atom compared with oxygen. Consequently, the hydrogen bond bridging the active-site nucleophile and Lys33 in WT CPs is broken with Cys1. Notably, the $2 F_{\mathrm{O}}-F_{\mathrm{C}}$ electron-density map of the T1C mutant also indicates that $\mathrm{Lys}_{3} 3 \mathrm{NH}_{2}$ is disordered. Together, these observations suggest that efficient peptide-bond hydrolysis requires that Lys $33 \mathrm{NH}_{2}$ hydrogen bonds to the active site nucleophile.

The benefit of Thr over Ser as the active-site nucleophile. All proteasomes strictly employ threonine as the active-site residue instead of serine. To investigate the reason for this singularity, we analysed a $\beta 5$-T1S mutant, which is viable but suffers from growth defects (Fig. 4a and Table 1). Activity assays with the 35-specific substrate Suc-LLVY-AMC demonstrated that the ChT-L activity of the T1S mutant is reduced by $40-45 \%$ compared with WT proteasomes depending on the incubation temperature (Fig. $4 \mathrm{~b}$ and Supplementary Fig. 9c). By contrast, turnover of the substrate Z-GGL-pNA, used to monitor ChT-L activity in situ but in a less quantitative fashion, is not detectably impaired (Supplementary Fig. 9a). Crystal structure analysis of the $\beta 5$-T1S mutant confirmed precursor processing (Fig. 4g), and ligand-complex structures with bortezomib and carfilzomib unambiguously corroborated the reactivity of Ser1 (Fig. 5).

However, the apo crystal structure revealed that $\operatorname{Ser} 1 \mathrm{O}^{\gamma}$ is turned away from the substrate-binding channel (Fig. 4g). Compared with $\mathrm{Thr}^{\gamma} \mathrm{O}^{\gamma}$ in WT CP structures, Ser1O ${ }^{\gamma}$ is rotated by $60^{\circ}$. This renders it unavailable for direct nucleophilic attack onto incoming substrates and first requires its reorientation, which is expected to delay substrate turnover. Because both conformations of Ser1O ${ }^{\gamma}$ are hydrogen-bonded to $\mathrm{Lys}_{3} 3 \mathrm{NH}_{2}$ (Fig. 4h), the relay system is capable of hydrolysing peptide substrates, albeit at lower rates compared with Thr1. The active-site residue Thrl is fixed in its position, as its methyl group is engaged in hydrophobic interactions with Thr3 
a
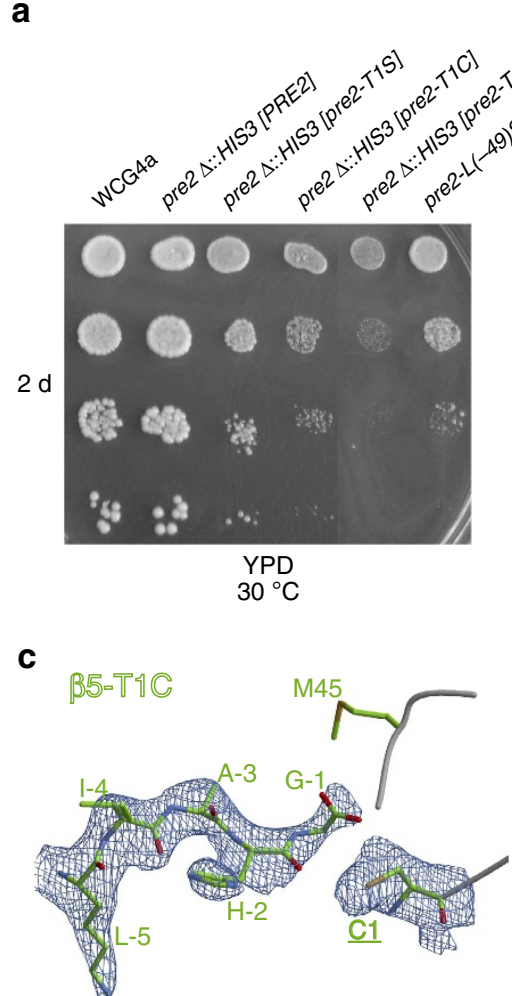

f $\beta 5$

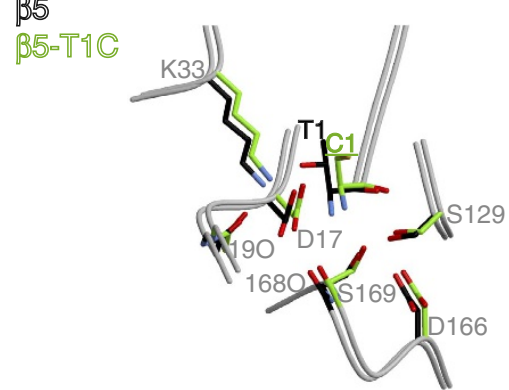

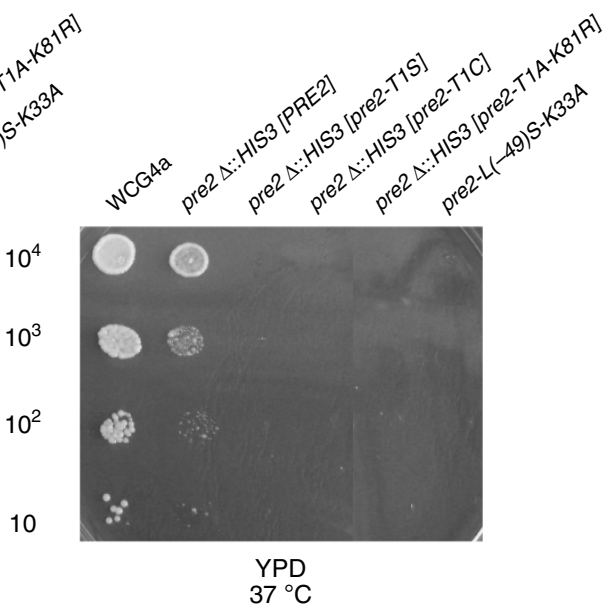

d

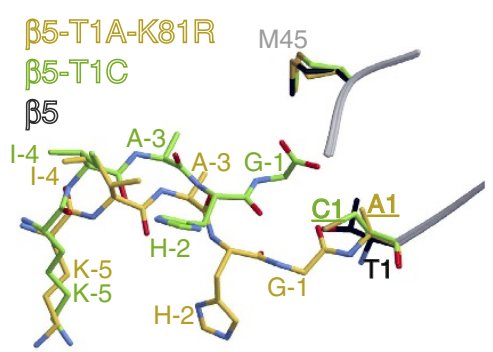

g

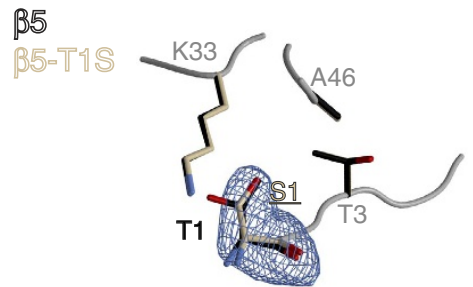

b

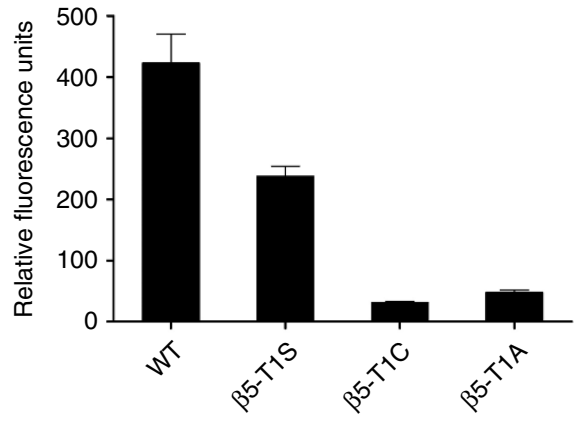

e

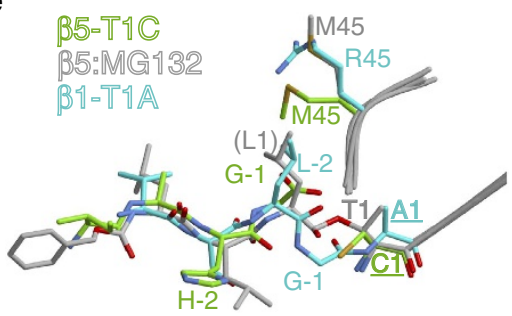

$\mathbf{h}$

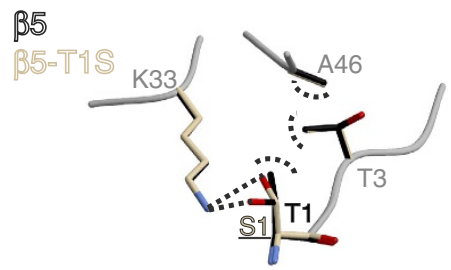

Figure 4 | The proteasome favours threonine as the active-site nucleophile. (a) Growth tests by serial dilution of WT and pre2 ( $\beta 5$ ) mutant yeast cultures reveal growth defects of the active-site mutants under the indicated conditions after 2 days $(2 \mathrm{~d}$ ) of incubation. (b) Purified WT and mutant proteasomes were tested for their chymotrypsin-like activity ( $\beta 5)$ using the substrate Suc-LLVY-AMC. Relative fluorescence units were measured in triplicate after $1 \mathrm{~h}$ of incubation at room temperature and are given as mean values. S.d.'s are indicated by error bars. (c) Illustration of the $2 F_{\mathrm{O}}-F_{\mathrm{C}}$ electron-density map (blue mesh contoured at $1 \sigma$ ) for the $35-\mathrm{T} 1 \mathrm{C}$ propeptide fragment. The prosegment is cleaved but still bound in the substrate-binding channel. Notably, His $(-2)$ does not occupy the S1 pocket formed by Met45, similar to what was observed for the $35-$ T1A-K81R mutant. (d) Structural superposition of the $\beta 5-T 1 A-$ K81R and the $\beta 5-T 1 C$ mutant subunits onto the WT $\beta 5$ subunit. (e) Structural superposition of the $\beta 5-\mathrm{T} 1 \mathrm{C}$ propeptide onto the $\beta 1-\mathrm{T} 1 \mathrm{~A}$ active site (blue) and the WT $\beta 5$ active site in complex with the proteasome inhibitor MG132 (ref. 30). The inhibitor as well as the propeptides adopt similar conformations in the substrate-binding channel. (f) Structural superposition of the WT $\beta 5$ and $\beta 5-T 1 C$ mutant active sites illustrates the different orientations of the hydroxyl group of Thr1 and the thiol side chain of Cys1. The SH group is rotated by $74^{\circ}$ compared with the $\mathrm{OH}$ group. ( $\mathrm{g}$ ) Structural superposition of the WT $\beta 5$ and $\beta 5$-T1S mutant active sites reveals different orientations of the hydroxyl groups of Thr1 and Ser1, respectively. The $2 F_{\mathrm{O}}-F_{\mathrm{C}}$ electron-density map for Ser1 (blue mesh contoured at $1 \sigma$ ) is illustrated. (h) The methyl group of Thr1 is anchored by hydrophobic interactions with Ala $46 C^{\beta}$ and Thr3 $C^{\gamma}$. Ser1 lacks this stabilization and is therefore rotated by $60^{\circ}$.

and Ala46 (Fig. 4h). Consequently, the hydroxyl group of Thr1 requires no reorientation before substrate cleavage and is thus more catalytically efficient than Ser1. In agreement, at an elevated growing temperature of $37^{\circ} \mathrm{C}$ the T1S mutant is unable to grow (Fig. 4a). In vitro, the mutant proteasome is less susceptible to proteasome inhibition by bortezomib (3.7-fold) and carfilzomib (1.8-fold; Fig. 5). Nevertheless, inhibitor complex structures indicate identical binding modes compared with the WT yCP structures, with the same inhibitors ${ }^{22,23}$. Notably, the affinity of the tetrapeptide carfilzomib is less impaired, as it is better stabilized in the substrate-binding channel than the dipeptide bortezomib, which lacks a defined P3 site and has only a few interactions with the surrounding protein. Hence, the mean residence time of carfilzomib at the active site is prolonged and the probability to covalently react with Ser1 is increased. Considered together, these results provide a plausible explanation for the invariance of threonine as the active-site nucleophile in proteasomes in all three domains of life, as well as in proteasome-like proteases such as HslV (ref. 24). 

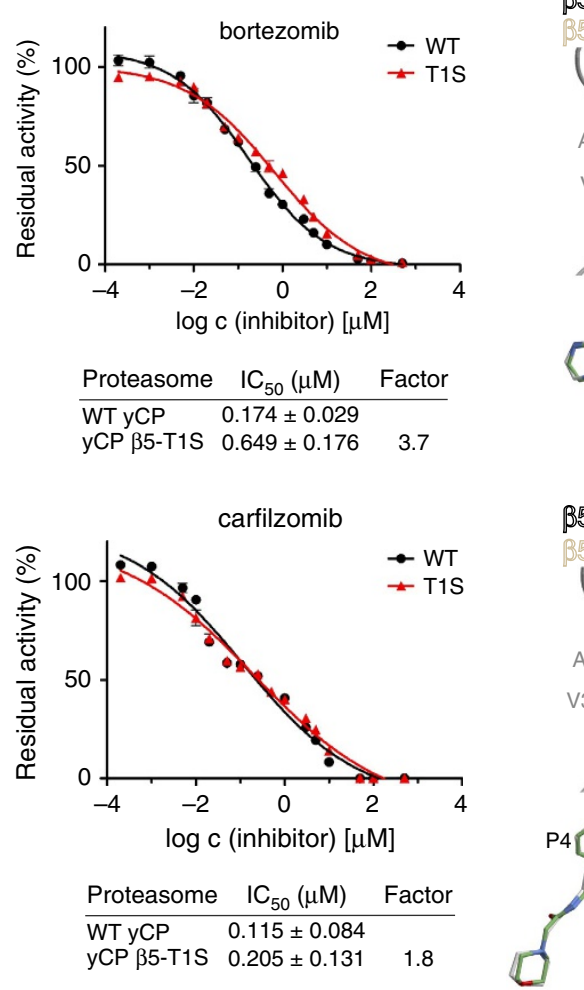
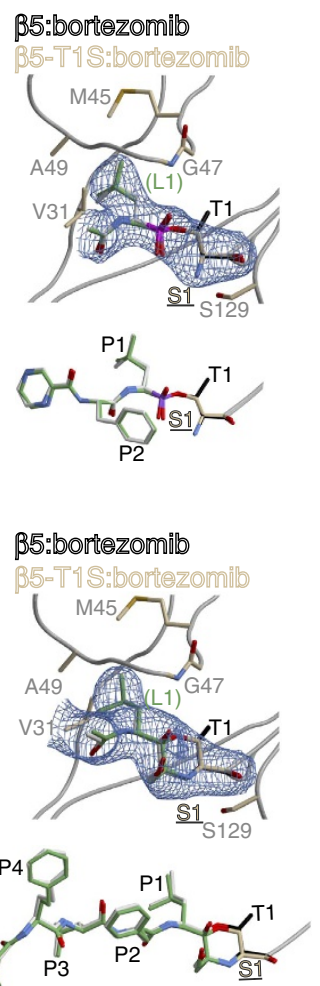
$\begin{array}{lll}\text { WT yCP } & 0.115 \pm 0.084 & \\ \text { yCP } \beta 5-T 1 S & 0.205 \pm 0.131 & 1.8\end{array}$

Figure 5 | Inhibition of WT and mutant $\beta$ 5-T1S proteasomes by bortezomib and carfilzomib. Inhibition assays (left panel). Purified yeast proteasomes were tested for the susceptibility of their ChT-L ( $\beta 5$ ) activity to inhibition by bortezomib and carfilzomib using the substrate Suc-LLVYAMC. $I C_{50}$ values were determined in triplicate; s.d.'s are indicated by error bars. Note that $I C_{50}$ values depend on time and enzyme concentration. Proteasomes (final concentration: $66 \mathrm{nM}$ ) were incubated with inhibitor for 45 min before substrate addition (final concentration: $200 \mu M$ ). Structures of the $\beta 5$-T1S mutant in complex with both ligands (green) prove the reactivity of Ser1 (right panel). The $2 F_{\mathrm{O}}-F_{\mathrm{C}}$ electron-density maps (blue mesh) for Ser1 (brown) and the covalently bound ligands (green; only the P1 site (Leu1) is shown) are contoured at $1 \sigma$. The WT proteasome:inhibitor complex structures (inhibitor in grey; Thr1 in black) are superimposed and demonstrate that mutation of Thr1 to Ser does not affect the binding mode of bortezomib or carfilzomib.

\section{Discussion}

The $20 \mathrm{~S}$ proteasome CP is the major non-lysosomal protease in eukaryotic cells, and its assembly is highly organized. The $\beta$-subunit propeptides, particularly that of $\beta 5$, are key factors that help drive proper assembly of the CP complex ${ }^{1}$. In addition, they prevent irreversible inactivation of the Thr1 $\mathrm{N}$ terminus by $N$-acetylation ${ }^{4,15,16}$. By contrast, the prosegments of $\beta$ subunits are dispensable for archaeal proteasome assembly, at least when heterologously expressed in Escherichia coli ${ }^{25}$. In eukaryotes, deletion of or failure to cleave the $\beta 1$ and $\beta 2$ propeptides is well tolerated $^{5,13-16}$. However, removal of the $\beta 5$ prosegment or any interference with its cleavage causes severe phenotypic defects ${ }^{1,13}$. These observations highlight the unique function and importance of the $\beta 5$ propeptide as well as the $\beta 5$ active site for maturation and function of the eukaryotic CP.

Here we have described the atomic structures of various $\beta 5$-T1A mutants, which allowed for the first time visualization of the residual $\beta 5$ propeptide. Depending on the $(-2)$ residue we observed various propeptide conformations, but Gly(-1) is in all structures perfectly located for the nucleophilic attack by $\mathrm{Thr} \mathrm{O}^{\gamma}$, although it does not adopt the tight turn observed for the prosegment of subunit $\beta 1$. From these data we conclude that only the positioning of Gly(-1) and Thr1 as well as the integrity of the proteasomal active site are required for autolysis. In this regard, inappropriate $\mathrm{N}$-acetylation of the Thr1 $\mathrm{N}$ terminus cannot be removed by $\mathrm{Thr} \mathrm{O}^{\gamma}$ due to the rotational freedom and flexibility of the acetyl group. The propeptide needs some anchoring in the substrate-binding channel to properly position Gly $(-1)$, but this seems to be independent of the orientation of residue $(-2)$.

Autolytic activation of the CP constitutes one of the final steps of proteasome biogenesis ${ }^{26}$, but the trigger for propeptide cleavage had remained enigmatic. On the basis of the numerous $\mathrm{CP}$ :ligand complexes solved during the past 18 years and in the current study, we provide a revised interpretation of proteasome active-site architecture. We propose a catalytic triad for the active site of the CP consisting of residues Thr1, Lys33 and Asp/Glu17, which are conserved among all proteolytically active eukaryotic, bacterial and archaeal proteasome subunits. $\mathrm{Lys} 33 \mathrm{NH}_{2}$ is expected to act as the proton acceptor during autocatalytic removal of the propeptides ${ }^{19}$, as well as during substrate proteolysis, while Asp $17 \mathrm{O}^{\delta}$ orients $\mathrm{Lys} 33 \mathrm{NH}_{2}$ and makes it more prone to protonation by raising its $\mathrm{p} K_{\mathrm{a}}$ (hydrogen bond distance: Lys $33 \mathrm{NH}_{3}^{+}-\mathrm{Asp} 17 \mathrm{O}^{\delta}: 2.9 \AA$ ). Analogously to the proteasome, a Thr-Lys-Asp triad is also found in L-asparaginase $\mathrm{e}^{27}$. Thus, specific protein surroundings can significantly alter the chemical properties of amino acids such as Lys to function as an acid-base catalyst ${ }^{28}$.

In this new view of the proteasomal active site, the positively charged $\mathrm{Thr} 1 \mathrm{NH}_{3}^{+}$-terminus hydrogen bonds to the amide nitrogen of incoming peptide substrates and stabilizes as well as activates them for the endoproteolytic cleavage by $\mathrm{Thr}^{\gamma} \mathrm{O}^{\gamma}$ (Fig. 3d). Consistent with this model, the positively charged Thr1 $\mathrm{N}$ terminus is engaged in hydrogen bonds with inhibitory compounds like fellutamide B (ref. 29), $\alpha$-ketoamides ${ }^{30}$, homobelactosin C (ref. 31) and salinosporamide A (ref. 32). Furthermore, opening of the $\beta$-lactone compound omuralide ${ }^{2}$ by Thr1 creates a C3-hydroxyl group, whose proton originates from Thr $1 \mathrm{NH}_{3}^{+}$. The resulting uncharged Thr $1 \mathrm{NH}_{2}$ is hydrogenbridged to the $\mathrm{C} 3-\mathrm{OH}$ group. In agreement, acetylation of the Thr1 $\mathrm{N}$ terminus irreversibly blocks hydrolytic activity ${ }^{15,16}$, and binding of substrates is prevented for steric reasons. By acting as a proton donor during catalysis, the Thr1 $\mathrm{N}$ terminus may also favour cleavage of substrate peptide bonds (Fig. 3d). In all proteases, collapse of the tetrahedral transition state results in selective breakage of the substrate amide bond, while the covalent interaction between the substrate and the enzyme persists. Cleavage of the scissile peptide bond requires protonation of the emerging free amine, and in the proteasome, the Thrl amine group is likely to assume this function. Analogously, $\mathrm{Thr}_{1} \mathrm{NH}_{3}^{+}$ might promote the bivalent reaction mode of epoxyketone inhibitors by protonating the epoxide moiety to create a positively charged trivalent oxygen atom that is subsequently nucleophilically attacked by $\mathrm{Thr} 1 \mathrm{NH}_{2}$.

During autolysis the Thr1 $\mathrm{N}$ terminus is engaged in a hydroxyoxazolidine ring intermediate (Fig. 3d), which is unstable and short-lived. Breakdown of this tetrahedral transition state releases the Thr1 $\mathrm{N}$ terminus that is protonated by aspartic acid 166 via Ser129OH to yield $\mathrm{Thr}_{1 \mathrm{NH}_{3}}{ }^{+}$. The residues Ser129 and Asp166 are expected to increase the $\mathrm{p} K_{\mathrm{a}}$ value of Thr1N, thereby favouring its charged state. Consistent with playing an essential role in proton shuttling, the mutation D166A prevents autolysis of the archaeal $\mathrm{CP}^{21}$ and the exchange D166N impairs catalytic activity of the yeast CP about $60 \%$. The mutation D166N lowers the $\mathrm{p} K_{\mathrm{a}}$ of $\mathrm{Thr} 1 \mathrm{~N}$, which is thus more likely to exist in the uncharged deprotonated state $\left(\mathrm{Thr} 1 \mathrm{NH}_{2}\right)$. This renders the $\mathrm{N}$ terminus less suitable to stabilize substrates and to protonate the first cleavage product during catalysis, although it favours its 
ability to act as a nucleophile. This interpretation agrees with the strongly reduced catalytic activity of the $\beta 5-\mathrm{D} 166 \mathrm{~N}$ mutant on the one hand, and the ability to react readily with carfilzomib on the other. Hence, the proteasome can be viewed as having a second triad that is essential for efficient proteolysis. While Lys $33 \mathrm{NH}_{2}$ and Asp $17 \mathrm{O}^{\delta}$ are required to deprotonate the Thr1 hydroxyl side chain, Ser129OH and Asp166OH serve to protonate the N-terminal amine group of Thr1.

In accord with the proposed Thr1-Lys33-Asp17 catalytic triad, crystallographic data on the proteolytically inactive $\beta 5$-T1C mutant demonstrate that the interaction of $\mathrm{Lys}_{3} 3 \mathrm{NH}_{2}$ and Cys1 is broken. Consequently, efficient substrate turnover or covalent modification by ligands is prevented. However, owing to Cys being a strong nucleophile, the propeptide can still be cleaved off over time. While only one single turnover is necessary for autolysis, continuous enzymatic activity is required for significant and detectable substrate hydrolysis. Notably, in the Ntn hydrolase penicillin acylase, substitution of the catalytic N-terminal Ser residue by Cys also inactivates the enzyme but still enables precursor processing ${ }^{33}$.

To investigate why the CP specifically employs threonine as its active-site residue, we used a 35 -T1S mutant of the yCP and characterized it biochemically and structurally. Activity assays with the $\beta 5$-T1S mutant revealed reduced turnover of Suc-LLVYAMC. We also observed slightly lower affinity of the $\beta 5$-T1S mutant $\mathrm{yCP}$ for the Food and Drug Administration-approved proteasome inhibitors bortezomib and carfilzomib. Structural analyses support these findings with the T1S mutant and provide an explanation for the strict use of Thr residues in proteasomes. Thr1 is well anchored in the active site by hydrophobic interactions of its $\mathrm{C}^{\gamma}$ methyl group with Ala46 $\left(\mathrm{C}^{\beta}\right)$, Lys33 (carbon side chain) and $\mathrm{Thr} 3\left(\mathrm{C}^{\gamma}\right)$. Notably, proteolytically active proteasome subunits from archaea, yeast and mammals, including constitutive, immuno- and thymoproteasome subunits, either encode Thr or Ile at position 3, indicating the importance of the $\mathrm{C}^{\gamma}$ for fixing the position of the nucleophilic Thr1. In contrast to Thr1, the hydroxyl group of Ser1 occupies the position of the Thr1 methyl side chain in the WT enzyme, which requires its reorientation relative to the substrate to allow cleavage (Fig. 4g,h). Notably, in the threonine aspartase Taspase1, mutation of the active-site Thr234 to Ser also places the side chain in the position of the methyl group of Thr234 in the WT, thereby reducing catalytic activity ${ }^{34}$. Similarly, although the serine mutant is active, threonine is more efficient in the context of the proteasome active site. The greater suitability of threonine for the proteasome active site, which has been noted in biochemical as well as in kinetic studies $^{35}$, constitutes a likely reason for the conservation of the Thr1 residue in all proteasomes from bacteria to eukaryotes.

\section{Methods}

Yeast mutagenesis. Site-directed mutagenesis was performed by standard techniques using oligonucleotides listed in Supplementary Table 2. The pre2/doa3 ( $\beta 5$ ) mutant alleles in the centromeric, TRP1- or LEU2-marked shuttle vectors YCplac22 and pRS315, respectively, were verified by sequencing and subsequently introduced into the yeast strains MHY784 (ref. 1) or YWH20 (ref. 13), which express WT PRE2 from a URA3-marked plasmid. Counter-selection against the URA3 marker with 5-fluoroorotic acid yielded strains expressing only the mutant forms of $\beta 5$.

The strain producing a processed $\beta 5-\mathrm{T} 1 \mathrm{~A}$ variant and the $\beta 5$ propeptide in trans is a derivative of YWH212 (ref. 15). It carries an additional deletion of the $N A T 1$ gene to avoid $N$-acetylation of Ala1; this strain exhibits extremely slow growth rates and served for crystallographic analysis only. All strains used in this study are listed in Supplementary Table 3.

Purification of yeast proteasomes. Yeast strains were grown in 18-1 cultures at $30^{\circ} \mathrm{C}$ in YPD into early stationary phase, and the yCPs were purified according to published procedures ${ }^{36}$. In brief, $120 \mathrm{~g}$ yeast cells were solubilized in $150 \mathrm{ml}$ of $50 \mathrm{mM} \mathrm{KH}{ }_{2} \mathrm{PO}_{4} / \mathrm{K}_{2} \mathrm{HPO}_{4}$ buffer ( $\mathrm{pH} 7.5$ ) and disrupted with a French press. Cell debris were removed by centrifugation for $30 \mathrm{~min}$ at 21,000 r.p.m. $\left(4{ }^{\circ} \mathrm{C}\right)$. The resulting supernatant was filtered and ammonium sulfate (saturated solution) was added to a final concentration of $30 \%(\mathrm{v} / \mathrm{v})$. This solution was loaded on a Phenyl Sepharose 6 Fast Flow column (GE Healthcare) pre-equilibrated with $1 \mathrm{M}$ ammonium sulfate in $20 \mathrm{mM} \mathrm{KH} \mathrm{PO}_{4} / \mathrm{K}_{2} \mathrm{HPO}_{4}$ ( $\mathrm{pH}$ 7.5). CPs were eluted by applying a linear gradient from 1 to $0 \mathrm{M}$ ammonium sulfate. Proteasomecontaining fractions were pooled and loaded onto a hydroxyapatite column (Bio-Rad) equilibrated with $20 \mathrm{mM} \mathrm{KH} \mathrm{PO}_{4} / \mathrm{K}_{2} \mathrm{HPO}_{4}(\mathrm{pH} 7.5)$. Elution of the CPs was achieved by increasing the phosphate buffer concentration from 20 to $500 \mathrm{mM}$. Anion-exchange chromatogaphy (Resource Q column (GE Healthcare), elution gradient from 0 to $500 \mathrm{mM}$ sodium chloride in $20 \mathrm{mM}$ Tris- $\mathrm{HCl}(\mathrm{pH} \mathrm{7.5)})$ and subsequent size-exclusion chromatography (Superose 6 10/300 GL (GE

Healthcare), $20 \mathrm{mM}$ Tris- $\mathrm{HCl}(\mathrm{pH} 7.5)$ and $150 \mathrm{mM} \mathrm{NaCl}$ ) resulted in pure CPs for crystallization and activity assays.

Fluorescence-based activity assay. ChT-L ( $\beta 5)$ activity of CPs was monitored by fluorescence spectroscopy using the model substrate Suc-LLVY-AMC. Purified yCPs $(66 \mathrm{nM}$ in $100 \mathrm{mM}$ Tris- $\mathrm{HCl}, \mathrm{pH} 7.5)$ were incubated with $300 \mu \mathrm{M}$ substrate for $1 \mathrm{~h}$ at room temperature or $37^{\circ} \mathrm{C}$. The reactions were stopped by diluting samples 1:10 in $20 \mathrm{mM}$ Tris- $\mathrm{HCl}, \mathrm{pH}$ 7.5. AMC fluorophores released by proteasomal activity were measured in triplicate with a Varian Cary Eclipse Fluorescence Spectrophotometer (Agilent Technologies) at $\lambda \mathrm{exc}=360 \mathrm{~nm}$ and $\lambda \mathrm{em}=460 \mathrm{~nm}$.

Inhibition assays. Purified yCPs were mixed with dimethylsulfoxide as a control or serial dilutions of inhibitor and incubated for $45 \mathrm{~min}$ at room temperature. A final concentration of yCP of $66 \mathrm{nM}$ was used. After addition of the peptide substrate Suc-LLVY-AMC to a final concentration of $200 \mu \mathrm{M}$ and incubation for $1 \mathrm{~h}$ at room temperature, the reaction was stopped by diluting the samples 1:10 in $20 \mathrm{mM}$ Tris- $\mathrm{HCl}, \mathrm{pH}$ 7.5. AMC fluorophores released by residual proteasomal activity were measured in triplicate at $\lambda \mathrm{exc}=360 \mathrm{~nm}$ and $\lambda \mathrm{em}=460 \mathrm{~nm}$. Relative fluorescence units were normalized to the dimethylsulfoxide-treated control. The calculated residual activities were plotted against the logarithm of the applied inhibitor concentration and fitted with GraphPad Prism 5. The IC50 value, the ligand concentration that leads to $50 \%$ inhibition of the enzymatic activity, was deduced from the fitted data.

Crystallization and structure determination. Mutant yCPs were crystallized as previously described for WT $20 \mathrm{~S}$ proteasomes ${ }^{36,37}$. Crystals were grown at $20^{\circ} \mathrm{C}$ using the hanging drop vapour diffusion method. Drops contained a 1:1 mixture of protein $\left(40 \mathrm{mg} \mathrm{ml}^{-1}\right)$ and reservoir solution $(25 \mathrm{mM}$ magnesium acetate, $100 \mathrm{mM}$ 2-(N-morpholino)ethanesulfonic acid (MES; pH 6.8) and 9-13\% (v/v) 2-methyl2,4-pentanediol (MPD)). Crystals were cryoprotected by addition of $5 \mu \mathrm{l}$ cryobuffer (20 mM magnesium acetate, $100 \mathrm{mM}$ MES, $\mathrm{pH}$ 6.8, and 30\% (v/v) MPD). Inhibitor complex structures were obtained by incubating crystals in $5 \mu \mathrm{l}$ cryobuffer supplemented with bortezomib or carfilzomib at a final concentration of $1.5 \mathrm{mM}$ for at least $8 \mathrm{~h}$.

Diffraction data were collected at the beamline X06SA at the Paul Scherrer Institute, SLS, Villigen, Switzerland $(\lambda=1.0 \AA)$. Evaluation of reflection intensities and data reduction were performed with the programme package $\mathrm{XDS}^{38}$. Molecular replacement was carried out with the coordinates of the yeast $20 \mathrm{~S}$ proteasome (PDB entry code: $5 \mathrm{CZ4}$ ) by rigid body refinements (REFMAC5; ref. 39). MAIN ${ }^{40}$ and COOT $^{41}$ were used to build models. TLS (Translation/ Libration/Screw) refinements finally yielded excellent $R_{\text {work }}$ and $R_{\mathrm{free}}$, as well as root mean squared deviation bond and angle values. The coordinates, proven to have good stereochemistry from the Ramachandran plots, were deposited in the RCSB Protein Data Bank (Supplementary Table 1).

The coordinates for the yeast $20 \mathrm{~S}$ proteasome deposited under the entry code $1 \mathrm{RYP}$ do not represent the WT yCP but the double-mutant $\beta 5-\mathrm{K} 33 \mathrm{R} \beta 1-\mathrm{T} 1 \mathrm{~A}$. At the time of deposition (in 1997), these data were the best available on the yCP. As yCP structure determination has become routine today, and structure refinement procedures have significantly improved, we here provide coordinates for the WT yCP at $2.3 \AA$ resolution (PDB entry code: $5 \mathrm{CZ4}$ ). Furthermore, the structures of most mutant yCPs described in this work were determined in their apo and ligandbound states. For mutants with proteolytically inactive $\beta 5$ subunits, the best crystallographic data obtained are given. For ligands or propeptide segments that were only partially defined in the $2 F_{\mathrm{O}}-F_{\mathrm{C}}$ electron-density map the occupancy was reduced (for details see Supplementary Table 1).

\section{References}

1. Chen, P. \& Hochstrasser, M. Autocatalytic subunit processing couples active site formation in the $20 \mathrm{~S}$ proteasome to completion of assembly. Cell $\mathbf{8 6}$, 961-972 (1996).

2. Groll, M. et al. Structure of $20 \mathrm{~S}$ proteasome from yeast at $2.4 \AA$ resolution. Nature 386, 463-471 (1997).

3. Krüger, E., Kloetzel, P. M. \& Enenkel, C. 20 S proteasome biogenesis. Biochimie 83, 289-293 (2001).

4. Groll, M. et al. The catalytic sites of $20 \mathrm{~S}$ proteasomes and their role in subunit maturation: a mutational and crystallographic study. Proc. Natl Acad. Sci. USA 96, 10976-10983 (1999). 
5. Ditzel, L. et al. Conformational constraints for protein self-cleavage in the proteasome. J. Mol. Biol. 279, 1187-1191 (1998).

6. Huber, E. M. et al. Immuno- and constitutive proteasome crystal structures reveal differences in substrate and inhibitor specificity. Cell 148, 727-738 (2012)

7. Huber, E. M. et al. Systematic analyses of substrate preferences of $20 \mathrm{~S}$ proteasomes using peptidic epoxyketone inhibitors. J. Am. Chem. Soc. 137, 7835-7842 (2015)

8. Marques, A. J., Palanimurugan, R., Matias, A. C., Ramos, P. C. \& Dohmen, R. J. Catalytic mechanism and assembly of the proteasome. Chem. Rev. 109, 1509-1536 (2009)

9. Löwe, J. et al. Crystal structure of the $20 \mathrm{~S}$ proteasome from the archaeon $T$. acidophilum at $3.4 \AA$ resolution. Science 268, 533-539 (1995).

10. Brannigan, J. A. et al. A protein catalytic framework with an N-terminal nucleophile is capable of self-activation. Nature 378, 416-419 (1995).

11. Seemüller, E. et al. Proteasome from Thermoplasma acidophilum: a threonine protease. Science 268, 579-582 (1995).

12. Dick, T. P. et al. Contribution of proteasomal beta-subunits to the cleavage of peptide substrates analyzed with yeast mutants. J. Biol. Chem. 273, 25637-25646 (1998)

13. Heinemeyer, W., Fischer, M., Krimmer, T., Stachon, U. \& Wolf, D. H. The active sites of the eukaryotic $20 \mathrm{~S}$ proteasome and their involvement in subunit precursor processing. J. Biol. Chem. 272, 25200-25209 (1997).

14. Arendt, C. S. \& Hochstrasser, M. Identification of the yeast 20 S proteasome catalytic centers and subunit interactions required for active-site formation. Proc. Natl Acad. Sci. USA 94, 7156-7161 (1997).

15. Jäger, S., Groll, M., Huber, R., Wolf, D. H. \& Heinemeyer, W. Proteasome betatype subunits: unequal roles of propeptides in core particle maturation and a hierarchy of active site function. J. Mol. Biol. 291, 997-1013 (1999).

16. Arendt, C. S. \& Hochstrasser, M. Eukaryotic 20 S proteasome catalytic subunit propeptides prevent active site inactivation by $\mathrm{N}$-terminal acetylation and promote particle assembly. EMBO J. 18, 3575-3585 (1999).

17. Polgar, L. The catalytic triad of serine peptidases. Cell. Mol. Life Sci. 62, 2161-2172 (2005)

18. Li, X., Li, Y., Arendt, C. S. \& Hochstrasser, M. Distinct elements in the proteasomal beta 5 subunit propeptide required for autocatalytic processing and proteasome assembly. J. Biol. Chem. 291, 1991-2003 (2015).

19. Schmidtke, G. et al. Analysis of mammalian $20 \mathrm{~S}$ proteasome biogenesis: the maturation of beta-subunits is an ordered two-step mechanism involving autocatalysis. EMBO J. 15, 6887-6898 (1996).

20. Wlodawer, A. Proteasome: a complex protease with a new fold and a distinct mechanism. Structure 3, 417-420 (1995).

21. Seemüller, E., Lupas, A. \& Baumeister, W. Autocatalytic processing of the $20 \mathrm{~S}$ proteasome. Nature 382, 468-471 (1996).

22. Groll, M., Berkers, C. R., Ploegh, H. L. \& Ovaa, H. Crystal structure of the boronic acid-based proteasome inhibitor bortezomib in complex with the yeast 20 S proteasome. Structure 14, 451-456 (2006).

23. Huber, E. M., Heinemeyer, W. \& Groll, M. Bortezomib-resistant mutant proteasomes: structural and biochemical evaluation with carfilzomib and ONX 0914. Structure 23, 407-417 (2015).

24. Bochtler, M., Ditzel, L., Groll, M. \& Huber, R. Crystal structure of heat shock locus V (HslV) from Escherichia coli. Proc. Natl Acad. Sci. USA 94, 6070-6074 (1997).

25. Zwickl, P., Kleinz, J. \& Baumeister, W. Critical elements in proteasome assembly. Nat. Struct. Biol. 1, 765-770 (1994)

26. Groll, M., Brandstetter, H., Bartunik, H., Bourenkow, G. \& Huber, R. Investigations on the maturation and regulation of archaebacterial proteasomes. J. Mol. Biol. 327, 75-83 (2003).

27. Lubkowski, J., Dauter, M., Aghaiypour, K., Wlodawer, A. \& Dauter, Z. Atomic resolution structure of Erwinia chrysanthemi L-asparaginase. Acta Crystallogr. D Biol. Crystallogr. 59, 84-92 (2003).

28. Gutteridge, A. \& Thornton, J. M. Understanding nature's catalytic toolkit. Trends Biochem. Sci. 30, 622-629 (2005).

29. Hines, J., Groll, M., Fahnestock, M. \& Crews, C. M. Proteasome inhibition by fellutamide B induces nerve growth factor synthesis. Chem. Biol. 15, 501-512 (2008).

30. Stein, M. L. et al. Systematic comparison of peptidic proteasome inhibitors highlights the alpha-ketoamide electrophile as an auspicious reversible lead motif. Angew. Chem. Int. Ed. 53, 1679-1683 (2014).
31. Groll, M., Larionov, O. V., Huber, R. \& de Meijere, A. Inhibitor-binding mode of homobelactosin C to proteasomes: new insights into class I MHC ligand generation. Proc. Natl Acad. Sci. USA 103, 4576-4579 (2006).

32. Groll, M., Huber, R. \& Potts, B. C. Crystal structures of Salinosporamide A (NPI-0052) and B (NPI-0047) in complex with the 20S proteasome reveal important consequences of beta-lactone ring opening and a mechanism for irreversible binding. J. Am. Chem. Soc. 128, 5136-5141 (2006).

33. Choi, K. S., Kim, J. A. \& Kang, H. S. Effects of site-directed mutations on processing and activities of penicillin G acylase from Escherichia coli ATCC 11105. J. Bacteriol. 174, 6270-6276 (1992).

34. Khan, J. A., Dunn, B. M. \& Tong, L. Crystal structure of human Taspase1, a crucial protease regulating the function of MLL. Structure 13, 1443-1452 (2005).

35. Kisselev, A. F., Songyang, Z. \& Goldberg, A. L. Why does threonine, and not serine, function as the active site nucleophile in proteasomes? J. Biol. Chem. 275, 14831-14837 (2000)

36. Gallastegui, N. \& Groll, M. Analysing properties of proteasome inhibitors using kinetic and X-ray crystallographic studies. Methods Mol. Biol. 832, 373-390 (2012).

37. Groll, M. \& Huber, R. Purification, crystallization, and X-ray analysis of the yeast 20S proteasome. Methods Enzymol. 398, 329-336 (2005).

38. Kabsch, W. XDS. Acta Crystallogr. D Biol. Crystallogr. 66, 125-132 (2010).

39. Vagin, A. A. et al. REFMAC5 dictionary: organization of prior chemical knowledge and guidelines for its use. Acta Crystallogr. D Biol. Crystallogr. 60, 2184-2195 (2004).

40. Turk, D. MAIN software for density averaging, model building, structure refinement and validation. Acta Crystallogr. D Biol. Crystallogr. 69, 1342-1357 (2013).

41. Emsley, P., Lohkamp, B., Scott, W. G. \& Cowtan, K. Features and development of Coot. Acta Crystallogr. D Biol. Crystallogr. 66, 486-501 (2010).

\section{Acknowledgements}

The staff of the beamline X06SA at the Paul Scherrer Institute, Swiss Light Source, Villigen, Switzerland is acknowledged for assistance during data collection. The research leading to these results has received funding from the European Community's Seventh Framework Program (FP7/2007-2013) under BioStruct-X (grant agreement No. 283570) We are most grateful to Richard Feicht for the professional purification and crystallization of yeast proteasomes. This work was supported by the SFB 1035/A2 to M.G. as well as the U.S. National Institutes of Health grants GM046904 and GM083050 to M.H.

\section{Author contributions}

E.M.H., W.H., X.L., C.S.A. and M.H. created yeast mutants; E.M.H. and W.H. performed activity and growth assays; E.M.H. and M.G. collected and analysed X-ray data; E.M.H., M.H. and M.G. wrote the manuscript.

\section{Additional information}

Accession codes: Coordinates and structure factors have been deposited in the Protein Data Bank, www.pdb.org (for PDB entry codes see Supplementary Table 1).

Supplementary Information accompanies this paper at http://www.nature.com/ naturecommunications

Competing financial interests: The authors declare no competing financial interests

Reprints and permission information is available online at http://npg.nature.com/ reprintsandpermissions/

How to cite this article: Huber, E. M. et al. A unified mechanism for proteolysis and autocatalytic activation in the 20S proteasome. Nat. Commun. 7:10900 doi: 10.1038/ ncomms10900 (2016)

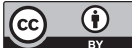

This work is licensed under a Creative Commons Attribution 4.0 International License. The images or other third party material in this article are included in the article's Creative Commons license, unless indicated otherwise in the credit line; if the material is not included under the Creative Commons license, users will need to obtain permission from the license holder to reproduce the material To view a copy of this license, visit http://creativecommons.org/licenses/by/4.0/ 